\title{
Antibiotic therapy affects functional behaviour in cystic fibrosis blood mononuclear cells
}

\section{To the Editor:}

Cystic fibrosis (CF) is the most common life-shortening genetic disorder in the Caucasian population and is due to mutations at the CF transmembrane conductance regulator (CFTR) gene leading to dysfunction of the protein, which normally acts as a chloride channel. This basic defect is associated with a progressive and lethal lung disease [1]. Opportunistic respiratory infections are common in CF patients' lungs, making antibiotics an important part of the regular care.

In the present study, we investigated whether a course of intravenous antibiotic treatment (10 days) for a pulmonary exacerbation in CF patients resulted in improved pulmonary function that was associated with changes in the expression of proteins forming the macromolecular complexes required for correct CFTR function in lymphomonocytes (LMNs).

While polymorphonuclear neutrophils (PMNs) preferentially accumulate on the CF surface epithelium, mononuclear cells are the predominant cell population in areas of cartilaginous destruction. The role of mononuclear cells in CF lung disease is presently poorly understood, although recently, a predominance of lymphocytes has been shown to infiltrate the subepithelial bronchial tissue from children with CF [2]. Lymphocytes found in the bronchial submucosa of CF subjects produce high levels of interleukin-17, a proinflammatory cytokine that regulates granulopoiesis and neutrophil recruitment [3].

17 nonsmoking subjects with CF who were homozygous for the F508del mutation (mean age 27.3 years, range 11-43 years), all of whom were pancreatic insufficient, were enrolled at the time of admission for a clinically diagnosed pulmonary exacerbation at the CF Regional Centre (Bari, Italy). The study was approved by the ethics committee of the Azienda Ospedaliera Universitaria "Policlinico" (Bari) (n.1373/CE/2012) and performed in accordance with the 1964 Declaration of Helsinki, after written informed consent was obtained from the adult study subjects, or the next of kin, caretakers, or guardians on behalf of the enrolled children.

Initial antibiotic choices (one, two to three antibiotics per patient) included azithromycin $(n=8)$, ciprofloxacin $(n=6)$, tobramycin $(n=5)$, ceftazidime $(n=4)$, minocycline $(n=2)$, meropenem $(n=1)$, levofloxacin $(n=1)$, sulfamethoxazole/trimethoprim $(n=1)$, amikacin $(n=1)$, imipenem $(n=1)$, teicoplanin $(\mathrm{n}=1)$ and linezolid $(\mathrm{n}=1)$. All treatments for acute exacerbation resulted in significant decreases in circulating PMNs (62.6\% of total white blood cell (WBC) count pre- versus $57.5 \%$ of total WBC count post-antibiotics, $\mathrm{p}=0.01)$, which paralleled the changes in serum C-reactive protein $\left(19.5 \mathrm{mg} \cdot \mathrm{dL}^{-1} \mathrm{pre}\right.$ - versus $11.3 \mathrm{mg} \cdot \mathrm{dL}^{-1}$ post-antibiotics, $\left.\mathrm{p}=0.02\right)$. The antibiotic treatment also resulted in a significant amelioration of forced expiratory volume in $1 \mathrm{~s}(47.2 \%$ predicted pre- versus $52.4 \%$ predicted post-antibiotics, $\mathrm{p}=0.04)$ and forced vital capacity (59.8\% predicted pre- versus $65.1 \%$ predicted post-antibiotics, $\mathrm{p}=0.04$ ).

Furthermore, during acute exacerbations, CF patients' LMNs had a severely impaired expression of the mature CFTR band with respect to LMNs from healthy subjects [4]. Here, in confirmation of that study, figure 1a shows a typical Western blot in which we loaded LMN lysates derived from a healthy donor and from a representative CF patient pre- and post-antibiotic treatment. In healthy LMNs, wild-type CFTR was expressed as the fully glycosylated mature form of the protein, band C $(180 \mathrm{kDa})$, and the core glycosylated form, band $\mathrm{B}$ $(160 \mathrm{kDa})$. As expected, in the CF LMNs pre-antibiotic treatment, F508delCFTR was almost completely expressed as the immature band $\mathrm{B}$, although a small amount of the mature band $\mathrm{C}$ protein was also observed in three of them. Antibiotic treatment resulted in the appearance of band $\mathrm{C}$, corresponding to a functional protein, as confirmed by spectrofluorimetric analysis using the chloride-sensitive dye, MQAE ( $N$-(ethoxycarbonylmethyl)-6-methoxyquinolinium bromide). CFTR-dependent chloride efflux was not detectable in LMNs from CF patients with acute exacerbation whereas antibiotic therapy significantly increased efflux to levels similar to those found in non-CF LMNs (figure 1b). The observed restoration of CFTR functionality was not associated with a statistically significant increase in CFTR mRNA levels post-antibiotic treatment (data not shown), which may therefore indicate that antibiotic treatment indirectly regulates the 
a)
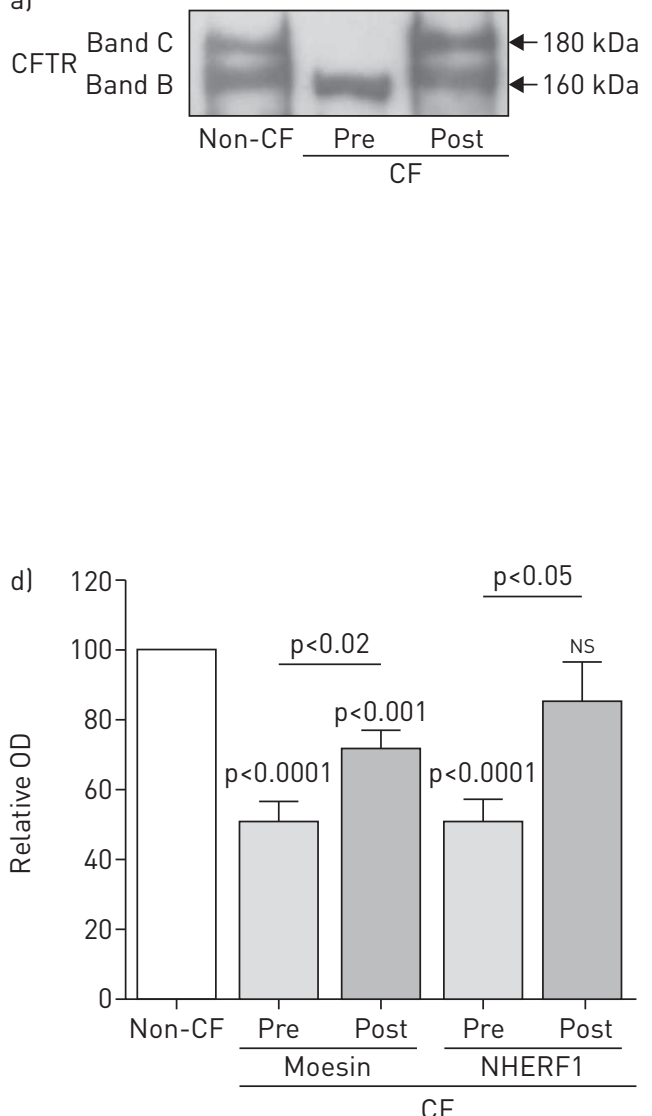

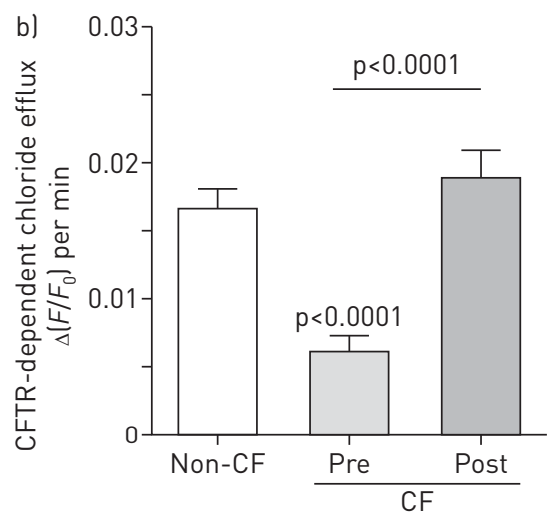

c)

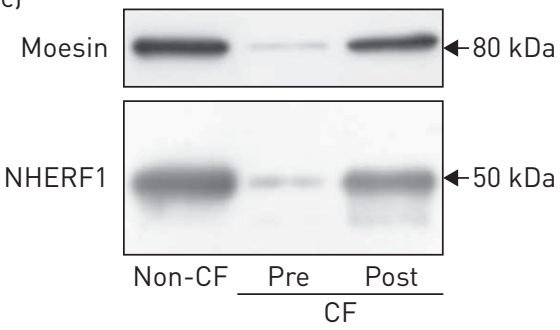

$\mathrm{p}<0.02$

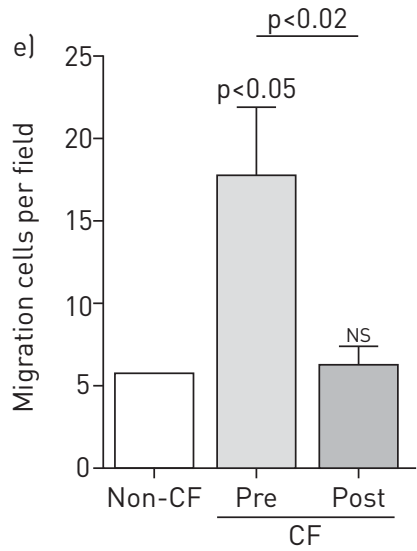

f)

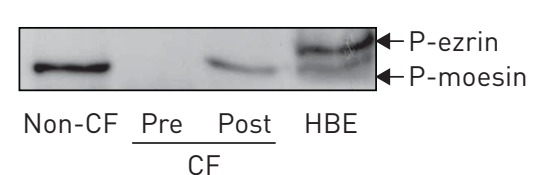

FIGURE 1 a) Cystic fibrosis transmembrane conductance regulator (CFTR) protein expression was analysed in lymphomonocyte (LMN) lysates from a healthy donor (non-CF) and a cystic fibrosis (CF) patient pre- and post-antibiotic therapy by Western blotting using an antibody against the C-terminus of human CFTR. b) LMNs seeded on $0.1 \%$ poly-L-lysine-coated glass coverslips were loaded overnight in culture medium containing $5 \mathrm{mM} \mathrm{N}$-(ethoxycarbonylmethyl)-6-methoxyquinolinium bromide (MQAE) at $37^{\circ} \mathrm{C}$ in a carbon dioxide incubator. The rate of chloride efflux induced by treatment with forskolin (10 $\left.\mu \mathrm{M}\right)$ plus IBMX (3-isobutyl-1-methylxanthine) $(100 \mu \mathrm{M})$ after substitution of chloride by nitrate in the perfusion medium was measured by the change in fluorescence of MQAE. CFTR activity was calculated as the difference in $(\Delta)$ the experimental fluorescence $(F)$ /maximum fluorescence $\left(F_{0}\right)$ ratio per minute obtained in the absence and presence of $\mathrm{CFTR}_{\mathrm{Inh}}-172(5 \mu \mathrm{M})$ in LMNs from non-CF and CF patients pre- and post-antibiotic therapy. c) Sodium/hydrogen exchanger regulatory factor (NHERF) 1 and moesin protein expression was analysed in LMNs by Western blotting using monoclonal anti-human NHERF1 antibody (dilution 1/250) and polyclonal anti-moesin antibody (dilution 1/5000). d) As the housekeeping proteins themselves were variably expressed, the total lane density of transferred proteins on the membrane stained with Ponceau red was used for the normalisation of proteins. The amount of both NHERF1 and moesin is summarised as the percentage of the change in the expression levels with respect to healthy LMNs. e) LMNs that had migrated across a polycarbonate membrane were stained and quantified by counting cells in at least 10 random fields per insert at 40× magnification using an inverted phase-contrast microscope. $\mathrm{f}$ ) Phospho (P)-moesin expression was analysed with a polyclonal anti-human phospho-ERM (ezrin (Thr567)/radixin (Thr564)/moesin (Thr558)) antibody (dilution 1/1000) in LMNs from non-CF and CF pre- and post-antibiotic therapy. Human bronchial epithelial (HBE) cells were used as positive control for the location of the P-moesin band. All the experiments were performed with 13 of the $17 \mathrm{CF}$ enrolled patients because less material was obtained from the other four patients. Nine healthy donors with similar sex and age were used as the control group. Statistical comparisons were made with respect to the values obtained in healthy LMNs, and between CF patients before and after antibiotic treatment. Bars represent the mean and whiskers represent the standard error of the mean. OD: optical density; ns: nonsignificant.

trafficking of F508delCFTR in the cell membrane via modulation of chaperones and cochaperones $(90-\mathrm{kDa}$ heat-shock protein (Hsp90)/70-kDa heat-shock protein (Hsp70)) known to bind CFTR [5].

F508delCFTR activity rescue was instead correlated to expression changes of the components of the multiprotein complex CFTR-sodium/hydrogen exchanger regulatory factor (NHERF)1-ERM (ezrin-radixinmoesin)-actin known to be involved in the regulation of CFTR localisation and activity [6]. Indeed, antibiotic therapy significantly increased NHERF1 and moesin protein expression levels (64\% and 45\%, respectively) compared with levels observed in acute exacerbation, which were significantly lower than healthy LMNs (figure 1c and 1d), in line with our previous observations in human bronchiolar epithelial cells [7, 8].

As ERM proteins have been also implicated in other aspects of lymphocyte activation, such as migration [9], we investigated whether the observed differences in moesin protein expression could correlate with altered migration in CF LMNs. Results from transwell migration studies showed that circulating LMNs obtained from CF patients with acute exacerbation had increased migration rates whereas migration returned to non-CF values post-antibiotic treatment (figure 1e). It is worth noting that our assay did not use chemotactic factors, thus suggesting that CF LMNs inherently display this migration property, i.e. they 
are pre-activated in the peripheral circulation. This "primed" status may result from the shedding of pro-inflammatory cytokines from the lung, where they are produced at high levels in CF exacerbations [10].

It is known that ERM function is partly controlled by phosphorylation on a conserved threonine in the actin-binding domain [11]. Moreover, it has been reported that, unlike in epithelial cells, ERM proteins occur predominantly in their active phosphorylated conformation in lymphocytes [12]. In line with these findings, we observed more ERM protein phosphorylation in healthy LMNs compared with CF LMNs, as well as phosphorylation levels in CF LMNs post-antibiotic therapy that were almost comparable to healthy LMNs (figure 1f). These results suggest a critical physiological role of ERM protein activation in lymphocytes. ERM protein dephosphorylation is known to be affected by chemokine signalling and leads to microvillar collapse in LMNs, thereby promoting the transition from tethering and rolling to integrin-dependent cell-cell adhesion [13, 14]. Thus, it is tempting to speculate that a more relaxed cytoskeleton, in response to reduced levels of activated moesin, may promote the increased LMN migration observed in the acute exacerbation condition.

Among the different antibiotics used in CF patients, azithromycin significantly activates chloride efflux in CF human bronchial epithelial cells $[15,16]$. Since this macrolide was used in many of the CF patients in this study, it could be, at least in part, responsible for the rescuing effect on CFTR and the macromolecular complex that allows correct positioning and function of CFTR on the plasma membrane.

In conclusion, we demonstrated, for the first time, that antibiotic treatment restores adequate levels of functional CFTR in LMNs, therefore contributing to improving the clinical status of CF patients. Our hypothesis is that antibiotic-mediated regulation of chaperones might allow increased folding and trafficking of mutant CFTR protein, thereby inducing partial CFTR function restoration. Despite the paucity of patients taken into consideration and the heterogeneity of antibiotic treatments, we found that their clinical status amelioration was consistently associated with increased functional expression of the defective CFTR chloride channel, increased expression of NHERF1 and moesin, and subsequent reduced LMN migration, probably suggesting that mononuclear cells may also take part in the inflammation-related damage of CF airways.

0

@ERSpublications

Antibiotic therapy indirectly restores adequate levels of functional F508delCFTR in CF lymphomonocytes http://ow.ly/Ncydg

Lorenzo Guerra ${ }^{1,5}$, Maria Favia ${ }^{1,5}$, Stefano Castellani ${ }^{2}$, Giovanna Barbuti ${ }^{3}$, Pasqualina Montemurro ${ }^{3}$, Anna Diana ${ }^{4}$, Teresa Santostasi ${ }^{4}$, Angela M. Polizzi ${ }^{4}$, Maria A. Mariggio ${ }^{3}$, Stephan J. Reshkin ${ }^{1}$, Antonio Manca ${ }^{4}$, Valeria Casavola ${ }^{1,6}$ and Massimo Conese ${ }^{2,6}$

${ }^{1}$ Department of Biosciences, Biotechnologies and Biopharmaceutics, University of Bari, Bari, Italy. ${ }^{2}$ Department of Medical and Surgical Sciences, University of Foggia, Foggia, Italy. ${ }^{3}$ Department of Biomedical Sciences and Human Oncology, Section of General Pathology, University of Bari, Bari, Italy. ${ }^{4}$ Cystic Fibrosis Regional Center, Department of Biomedical and Human Oncology, Section Pediatrics, U.O. "B. Trambusti", University of Bari, Bari, Italy. ${ }^{5}$ These authors contributed equally to this work. ${ }^{6}$ These authors share senior authorship.

Correspondence: Lorenzo Guerra, Department of Biosciences, Biotechnologies and Biopharmaceutics, University of Bari, Via Orabona 4, Bari 70126, Italy. E-mail: lorenzo.guerra1@uniba.it

Received: Oct 132014 | Accepted after revision: April 172015 | First published online: June 112015

Support statement: This work was supported by the Italian law 548/93 for the years 1999-2003, project "Study of factors which influence prognosis in cystic fibrosis".

Conflict of interest: None declared.

\section{References}

Rowe SM, Miller S, Sorscher EJ. Cystic fibrosis. N Engl J Med 2005; 352: 1992-2001.

2 Regamey N, Tsartsali L, Hilliard TN, et al. Distinct patterns of inflammation in the airway lumen and bronchial mucosa of children with cystic fibrosis. Thorax 2012; 67: 164-170.

3 Tan HL, Regamey, N Brown S, et al. The Th17 pathway in cystic fibrosis lung disease. Am J Respir Crit Care Med 2011; 184: 252-258.

4 Sorio C, Buffelli M, Angiari C, et al. Defective CFTR expression and function are detectable in blood monocytes: development of a new blood test for cystic fibrosis. PLoS One 2011; 6: e22212.

5 Wang X, Venable J, LaPointe P, et al. Hsp90 cochaperone Aha1 downregulation rescues misfolding of CFTR in cystic fibrosis. Cell 2006; 127: 803-815.

6 Harris BZ, Lim WA. Mechanism and role of PDZ domains in signaling complex assembly. J Cell Sci 2001; 114: 3219-3231.

7 Guerra L, Fanelli T, Favia M, et al. $\mathrm{Na}^{+} / \mathrm{H}^{+}$exchanger regulatory factor isoform 1 overexpression modulates cystic fibrosis transmembrane conductance regulator (CFTR) expression and activity in human airway $16 \mathrm{HBE} 14 \mathrm{o}^{-}$cells and rescues $\Delta$ F508 CFTR functional expression in cystic fibrosis cells. J Biol Chem 2005; 280: 40925-40933. 
8 Favia M, Guerra L, Fanelli T, et al. $\mathrm{Na}^{+} / \mathrm{H}^{+}$exchanger regulatory factor 1 overexpression-dependent increase of cytoskeleton organization is fundamental in the rescue of F508del cystic fibrosis transmembrane conductance regulator in human airway CFBE410 ${ }^{-}$cells. Mol Biol Cell 2010; 21: 73-86.

9 Brown MJ, Hallam JA, Colucci-Guyon E, et al. Rigidity of circulating lymphocytes is primarily conferred by vimentin intermediate filaments. J Immunol 2001; 166: 6640-6646.

10 Colombo C, Costantini D, Rocchi A, et al. Cytokine levels in sputum of cystic fibrosis patients before and after antibiotic therapy. Pediatr Pulmonol 2005; 40: 15-21.

11 Ivetic A, Deka J, Ridley A, et al. The cytoplasmic tail of L-selectin interacts with members of the Ezrin-Radixin-Moesin (ERM) family of proteins: cell activation-dependent binding of Moesin but not Ezrin. J Biol Chem 2002; 277: 2321-2329.

12 Parameswaran N, Gupta N. Re-defining ERM function in lymphocyte activation and migration. Immunol Rev 2013; 256: 63-79.

13 Brown MJ, Nijhara R, Hallam JA, et al. Chemokine stimulation of human peripheral blood T lymphocytes induces rapid dephosphorylation of ERM proteins, which facilitates loss of microvilli and polarization. Blood 2003; 102: 3890-3899.

14 Shaffer MH, Dupree RS, Zhu P, et al. Ezrin and moesin function together to promote T cell activation. J Immunol 2009; 182: 1021-1032.

15 Saint-Criq V, Rebeyrol C, Ruffin M, et al. Restoration of chloride efflux by azithromycin in airway epithelial cells of cystic fibrosis patients. Antimicrob Agents Chemother 2011; 55: 1792-1793.

16 Oliynyk I, Varelogianni G, Schalling M, et al. Azithromycin increases chloride efflux from cystic fibrosis airway epithelial cells. Exp Lung Res 2009; 35: 210-221.

\title{
The problem of early mortality in pneumococcal pneumonia: a study of risk factors
}

\author{
To the Editor:
}

The mortality of pneumococcal pneumonia, and especially the number deaths that occur soon after presentation, remains unacceptably high [1]. In 1964, AUSTRIAN and GolD [2] observed that 60\% of deaths in patients with invasive pneumococcal pneumonia (IPP) occurred within the first 5 days. Unfortunately, this does not appear to have changed over the subsequent five decades [3].

Different factors related to the mortality of pneumococcal disease have been described, including host factors such as age, comorbidities or immunosuppressive conditions [4], and organism-related factors such as serotype, bacterial load or viral co-infection $[3,5-7]$. These factors have all been primarily identified to predict overall mortality, but information regarding the determinants of early mortality is scarce. It has been hypothesised that early deaths are more likely to be due to an inappropriate inflammatory response triggered by Streptococcus pneumoniae than to the micro-organism itself [8]. Thus, the factors that influence the early mortality may differ from those associated with late mortality. We performed the present study in order to assess factors associated with early and late mortality in IPP.

This is a multicentre longitudinal study of adults hospitalised because of IPP in three hospitals in Catalonia, Spain. In these hospitals, all microbiological strains isolated in sterile samples are collected systematically. IPP was diagnosed when a patient had consistent clinical findings plus a new pulmonary infiltrate on chest radiography and isolation of $S$. pneumoniae in blood and/or pleural fluid cultures. The strains were serotyped by Quellung reaction and/or dot-blot assay. Serotypes were grouped according to high (serotypes 3,6A, 6B, 9N, 19A, 19F and 23F), medium (9V, 12F, 14 and 22F) and low $(1,4,5,7 \mathrm{~F}$ and 8) serotype-specific case fatality rates [6]. Antibiotic therapy was administered at the discretion of the attending physician and the hospital guidelines. Treatment was considered appropriate if at least one antibiotic administered during the first $48 \mathrm{~h}$ showed full sensitivity against the isolated strains. To identify the risk factors for early mortality (death within 5 days of admission), late mortality (hospital mortality $>5$ days after admission) and survival, a multinomial logistic regression analysis was performed.

Over the study period (from 1996 to 2013), 1588 consecutive adults with IPP were diagnosed. Overall, 221 patients (13.9\%) died in hospital. 121 (54.5\%) died in the first 5 days after admission; 80 (36.1\%) of them died in the first $48 \mathrm{~h}$. Patients with early mortality were older (mean age 67.1 years) and $65.5 \%$ had an 